\title{
Modeling of Free Radical Polymerization up to High Conversion. II. Development of a Mathematical Model
}

\author{
N. TEFERA, G. WEICKERT, K. R. WESTERTERP \\ Chemical Reaction Engineering Laboratories, Faculty of Chemical Technology, University of Twente, P.O. Box 217, \\ 7500 AE Enschede, The Netherlands
}

Received 6 October 1995; accepted 5 September 1996

\begin{abstract}
In free radical polymerization diffusion-controlled processes take place simultaneously to the normal chemical reactions. Despite extensive efforts to model such processes a consistent model for the design of a polymerization reactor has not yet been established. In this article a semiempirical model describing the conversion, polymerization degree, and molecular weight distribution (MWD) for the free radical polymerization is developed for the entire course of the reaction. The model includes the change of termination, propagation, transfer, and initiation rate. By simultaneous parameter estimation from the conversion and degrees of polymerization data the model parameters have been determined for isothermal polymerizations of methyl methacrylate (MMA) and styrene (ST). The simulation results for the conversion, degrees of polymerization, and MWD are in good accordance with experimental data for suspension and bulk polymerization of MMA and ST up to very high conversions. The influence of diffusion on the propagation rate in case of polymerization of MMA is negligible compared to the influence of the cage effect on the radical efficiency; in case of ST polymerization both effects must be included in the kinetic model. The model presented is also tested for polymerizations conducted in the presence of solvent and/or chain transfer agents. (C) 1997 John Wiley \& Sons, Inc. J Appl Polym Sci 63: 1663-1680, 1997
\end{abstract}

Key words: mathematical model; diffusion-controlled radical polymerization; highconversion modeling

\section{INTRODUCTION}

In part I of this series it was shown that existing high conversion models are not suited to describe both the polymerization degree and the conversion data simultaneously. ${ }^{1}$ Other models such as that of Soong et al. ${ }^{2}$ and Kiparissides et al. ${ }^{3}$ seems to be more relevant and advantageous. But as it was discussed in part I of this series, the Chui model does not consider the change of the radical

\footnotetext{
Correspondence to: K. R. Westerterp.

Contract grant sponsor: Friedrich-Naumann Foundation (Germany).
}

(C) 1997 John Wiley \& Sons, Inc. CCC 0021-8995/97/121663-18 efficiency and also contains the initial amount of initiator. Its application to other polymerization conditions, therefore, may not be possible. For example, in a semibatch polymerization with initiator feeding, the functional dependence of $k_{t}$ on the initial initiator concentration will not hold true. The parameters of the Kiparissides model have a physical meaning, but these are not available either for many monomer-polymer systems or for other components like solvents. Therefore, some of the model parameters should be determined empirically from other measurements. Also, Kiparissides' model does not describe the entire conversion range, especially in the region of high conversion, better than the other models. 


\section{THEORETICAL ASPECTS}

\section{Reaction Mechanism}

The reaction mechanism adopted in the model presented below consists of the well-known steps of initiation, propagation, termination, and transfer to monomer and to transfer agents. The various reactions taking place are

1. Initiation:

$$
\begin{gathered}
I \stackrel{k_{d}}{\rightarrow} 2 R \quad \text { homolytic scission } \\
R+M \stackrel{k_{i}}{\rightarrow} P_{1} \quad \text { radical initiation } \\
a M \stackrel{k_{i a}}{\rightarrow} 2 P_{1} \quad \text { thermal initiation }
\end{gathered}
$$

2. Propagation:

$$
P_{j}+M \stackrel{k_{p}}{\rightarrow} P_{j+1}
$$

3. Transfer:

$$
\begin{gathered}
P_{j}+M \stackrel{k_{t r, M}}{\rightarrow} M_{j}+P_{1} \quad \text { transfer to monomer } \\
P_{j}+S \stackrel{k_{t r, S}}{\rightarrow} M_{j}+P_{1} \quad \text { transfer to solvent } \\
\mathrm{P}_{j}+C \stackrel{k_{t r, C}}{\rightarrow} M_{j}+P_{1} \quad \text { transfer to transfer agent }
\end{gathered}
$$

4. Termination:

$$
\begin{array}{cl}
P_{j}+P_{k} \stackrel{k_{t c}}{\rightarrow} M_{j+k} & \text { recombination } \\
P_{j}+P_{k} \stackrel{k_{t d}}{\rightarrow} M_{j}+M_{k} & \text { disproportionation }
\end{array}
$$

where $I$ is initiator, $R$ primary initiator radical, $a$ the reaction order of the thermal initiation, $M$ monomer, $M_{j}$ dead polymer with degree of polymerization $j, P_{j}$ the corresponding growing polymer radical, and $k$ the relevant rate constants.

\section{Kinetic Modeling}

The detailed derivation of the corresponding kinetic models based on the above polymerization mechanism have been given elsewhere. ${ }^{1,18}$ Their derivation is based on the application of the method of moments to the mass balances for each of the species present in the polymerization mixture. For a well-stirred batch reactor-applying the long chain hypothesis LCH for the monomer consumption and the quasi-steady-state approximation QSSA for the radical concentrations-the following set of algebraic and nonlinear ordinary differential equations are obtained to describe the progress of the reaction and molecular weight development:

$$
\begin{aligned}
\frac{d Y_{I, i}}{d t} & =-k_{d, i} Y_{I, i} \\
\frac{d Y_{p}}{d t} & =R_{p} \\
\frac{d Y_{S}}{d t} & =-k_{t r, S} Y_{S} \lambda_{0} \\
\frac{d Y_{C}}{d t} & =-k_{t r, C} Y_{C} \lambda_{0} \\
\frac{d \overline{P_{n}}}{d t} & =\frac{\overline{P_{n}} R_{p}\left(P_{n}^{d}-\overline{P_{n}}\right)}{P_{n}^{d} Y_{p}} \\
\frac{d \bar{P}_{w}}{d t} & =\frac{R_{p}\left(P_{w}^{d}-\bar{P}_{w}\right)}{Y_{p}}
\end{aligned}
$$

In these equations, $Y_{j}$ represents the weight fraction of component $j, P_{n}$ and $P_{w}$ the number and weight average degrees of polymerization, an overbar the integrated or cumulative value, and the superscript $d$ the instantaneous value; $M_{M}$ and $\rho$ are the molecular weight of the monomer and the density of the polymerization mix, respectively. The instantaneous number-average and weight-average degree of polymerizations are given by

$$
\begin{aligned}
P_{n}^{d} & =\frac{\left(T^{*}+k_{t} \lambda_{0}\right) \lambda_{1}}{\left\{T^{*}+\left(k_{t d}+0.5 k_{t c}\right) \lambda_{0}\right\} \lambda_{0}} \\
P_{w}^{d} & =\frac{\left(T^{*}+k_{t} \lambda_{0}\right) \lambda_{2}+k_{t c} \lambda_{1}^{2}}{\left(T^{*}+k_{t} \lambda_{0}\right) \lambda_{1}}
\end{aligned}
$$

where $T^{*}$ denotes the total transfer term $T^{*}$ $=k_{t r, M} M+k_{t r, S} S+k_{t r, C} C$, and $\lambda_{0}, \lambda_{1}$, and $\lambda_{2}$ are the zeroth, first, and second moments, respectively, of the growing radicals; they can be calculated with the following equations:

$$
\begin{aligned}
& \lambda_{0}=\left(\frac{r_{g}}{k_{t}}\right)^{0.5} \\
& \lambda_{1}=\frac{r_{g}+\left(k_{P} M+T^{*}\right) \lambda_{0}}{T^{*}+k_{t} \lambda_{0}} \\
& \lambda_{2}=\frac{r_{g}+k_{P} M\left(2 \lambda_{1}+\lambda_{0}\right)+T^{*} \lambda_{0}}{T^{*}+k_{t} \lambda_{0}}
\end{aligned}
$$


Table I Numerical Values of Parameters Used in the Model Calculations

\begin{tabular}{llll}
\hline Parameter & \multicolumn{1}{c}{ Unit } & \multicolumn{1}{c}{ MMA-Polymerization $^{18-20}$} & \multicolumn{1}{c}{ ST-Polymerization $^{18-20}$} \\
\hline$k_{d}(\mathrm{AIBN})$ & $\mathrm{s}^{-1}$ & $2.8 \times 10^{15} \mathrm{exp}(-15685 / T)$ & $1.58 \times 10^{15} \exp (-15508 / T)$ \\
$f(\mathrm{AIBN})$ & - & 0.43 & $89.81 \times \exp (-1713 / T)$ \\
$k_{i a}$ & $1^{2} \mathrm{~mol}^{-2} \mathrm{~s}^{-1}$ & - & $2.19 \times 10^{5} \exp (-13809 / T)$ \\
$a$ & - & - & 3 \\
$k_{p}$ & $1 \mathrm{~mol}^{-1} \mathrm{~s}^{-1}$ & $4.9 \times 10^{5} \exp (-2190 / T)$ & $1.0213 \times 10^{7} \exp (-3557 / T)$ \\
$k_{t r, M}$ & $1 \mathrm{~mol}^{-1} \mathrm{~s}^{-1}$ & $2.324 \times 10^{8} \exp (-9218 / T)$ & $4.8307 \times 10^{6} \exp (-6480 / T)$ \\
$k_{t r, C}(\mathrm{DDM})$ & $1 \mathrm{~mol}^{-1} \mathrm{~s}^{-1}$ & $771\left(\mathrm{at} 70^{\circ} \mathrm{C}\right)$ & - \\
$k_{t}$ & $1 \mathrm{~mol}^{-1} \mathrm{~s}^{-1}$ & $9.8 \times 10^{7} \exp (-353 / T)$ & $1.2583 \times 10^{9} \exp (-844 / T)$ \\
$k_{t d} / k_{t}$ & - & 1 & 0.2 \\
$\rho_{M}$ & $\mathrm{~g} / \mathrm{L}$ & $968-1.15(T-273.15)$ & $924-0.918(T-273.15)$ \\
$\rho P$ & $\mathrm{~g} / \mathrm{L}$ & $1.212 \times 10^{3}-0.845(T-273.15)$ & 242.6 \\
$T_{g, M}$ & $\mathrm{~K}$ & 167 & 373 \\
$T_{g, P}$ & $\mathrm{~K}$ & 378 & $10^{-3}$ \\
$\alpha_{M}$ & $\mathrm{~K}^{-1}$ & $10^{-3}$ & $4.8 \times 10^{-4}-0.605(T-273.15)$ \\
$\alpha_{P}$ & $\mathrm{~K}$ & $4.8 \times 10^{-4}$ &
\end{tabular}

The rate of polymerization $R_{p}$ and the initiation rate $r_{g}$ are given by

$$
\begin{aligned}
R_{P} & =k_{P} M \lambda_{0} \frac{M_{M}}{\bar{\rho}} \\
r_{g} & =2 f k_{d} I+2 k_{i a} M^{a}
\end{aligned}
$$

The number- and weight-average molecular weights are obtained by multiplication of the corresponding polymerization degrees with the molecular weight of the monomer.

The simultaneous numerical solution of the above equations through the use of a standard library subroutine LSODA is a part of the parameter estimation program $\mathrm{P} 1$ available in program package "PolyReac ${ }^{\mathrm{e}}$." The details of this parameter estimation program and the mathematical methods have been reported elsewhere. ${ }^{21}$ The numerical values of the initial kinetic rate constants and the physical and transport properties of the MMA-PMMA and ST-PS system are reported in Table I.

\section{Development of the Model}

\section{Rate of Termination}

It is generally assumed that the termination step between two macro radicals involves a three-stage process. Initially, the two macro radicals diffuse toward one another by translation of the center of the macro radical so that certain segments of the chains are in contact; This is known as translational diffusion. This is followed by segmental diffusion and rearrangement of radical chain ends to make collision of radical ends possible. Only after the proper orientation of chain ends termination reaction can take place.

Analogous to most gel effect models our model development is also based on the above quantitative description of the termination reaction as a three-stage process. At the early stage of polymerization segmental diffusion or chemical reaction is the rate-determining step in the termination reaction, while at intermediate conversions the translational diffusion dominates the rate of termination. The reaction diffusion takes place parallel to all the above processes. The apparent termination rate constant $k_{t}$ can be expressed as ${ }^{4-8}$

$$
k_{t}=\frac{1}{\frac{1}{k_{t R}}+\frac{1}{k_{T D}^{*}}}+k_{R D}
$$

The molecular weight dependence of the translational diffusion coefficient can be approximated by the following expression:

$$
k_{T D}^{*}=k_{T D} / M_{w}^{n}
$$

Substitution of eq. (11b) into eq. (11a) gives

$$
k_{t}=\frac{1}{\frac{1}{k_{t R}}+\frac{M_{w}^{n}}{k_{T D}}}+k_{R D}
$$


where

$$
k_{T D}=D \exp \left(-\frac{g 1}{V_{f}}\right)
$$

Experimentally, we obtain the apparent $k_{t}$ instead of the intrinsic $k_{t R}$. Therefore, the intrinsic value of the termination constant $k_{t R}$ should be determined from the initial polymerization rate where the conversion $\mathrm{X}$ of monomers tends to zero. Applying the initial conditions in eq. (11) we obtain

$$
k_{t 0}=\frac{1}{\frac{1}{k_{t R}}+\frac{M_{w 0}^{n}}{k_{T D 0}}}+k_{R D 0}
$$

According to eq. (13) $k_{t 0}$ formally depends on the molecular weight of the dead polymer. At low conversions, this effect is negligible. Using eq. (13), the sought intrinsic termination constant $k_{t R}$ can be calculated according to eq. (14):

$$
\frac{1}{k_{t R}}=\frac{1}{k_{t 0}-k_{R D 0}}-\frac{M_{w 0}^{n}}{k_{T D 0}}
$$

Equation (12) is assumed to be true at $t=0$ :

$$
k_{T D 0}=D \exp \left(-\frac{g 1}{V_{f 0}}\right)
$$

After substitution of eqs. (12), (14), and (15) into eq. (11) and elimination of $D$, the following equation is obtained for the apparent termination constant $k_{t}$ :

$$
k_{t}=\frac{1}{\frac{1}{k_{t 0}-k_{R D 0}}+\frac{1}{k_{T D 0}}}+k_{R D}
$$

with

$$
X_{V f}=\frac{1}{V_{f}}-\frac{1}{V_{f 0}}
$$

As a result of eq. (13), numerical problems may arise from eq. (16) if the term in the parenthesis is negative. This can be true in some special cases where the $M_{w}$ is changing from high to low molec- ular weight within a few percent of monomer conversion. In these cases one should set $M_{w}=M_{w} 0$. This correction does not influence the final results dramatically because this behavior is only possible in the low conversion range where the influence of the molecular weight on $k_{t}$ is small.

The free volume $V_{f}$ was obtained assuming additivity of the free volume of the reaction components:

$$
V_{f}=\sum_{i} V_{f, i} \varphi_{i}
$$

$V_{f i}$ and $\varphi_{i}$ represent free volume and a volume fraction of the component $i$, respectively. The free volume for the component $i$ is given by Bueche ${ }^{8}$ : $V_{f, i}=V_{f, i 0}+a_{i}\left(T-T_{g, i}\right)$, where $V_{f i 0}=0.025$. In order to calculate the "reaction diffusion" or residual termination rate constant $k_{R D}$, Russell et al. ${ }^{9}$ proposed two different models based on the chain flexibility. Soh and Sundberg ${ }^{10}$ used the swept out volume theory to develop a model for $k_{R D}$. All models assume that $k_{R D}$ is proportional to the frequency of the monomer addition to the radical end. For the analogue we use the following equation to calculate $k_{R D}{ }^{5,7,9,10}$ :

$$
k_{R D}=A k_{P} C_{M}
$$

To determine the proportionality parameter $A$, Soh and Sundberg ${ }^{10}$ provide one single equation; on the other hand, Russell et al. ${ }^{9}$ derive two equations for the rigid and flexible chains, respectively. For the AIBN-initiated MMA polymerization at $70^{\circ} \mathrm{C}$ and initiator concentration $0.01548 \mathrm{~mol} / \mathrm{L}$ the Soh and Sundberg equation gives 5.85, and those of Russell et al. 0.703 and 9.35 for the rigid and flexible chains.

In the present work the value of $A$ is set equal to 1 after investigation of experimental data. We find a good agreement between model and experiment for other reaction systems also.

The diffusion term in the initial stage of the polymerization has no effect on the conversion or on the average degrees of polymerization $P_{n}$ and $P_{w}$. The use of the initial values in eq. (16) is necessary for the mathematical handling of the differential equations in the model simulation. Note that it is also possible to formulate other initial conditions, in that case the values of the fitted parameters may be different.

\section{Rate of Propagation}

At very high conversions even movement of small molecules is restricted. As a result the propaga- 
tion reaction becomes diffusion controlled. The apparent propagation rate constant $k_{p}$ is now expressed in terms of the intrinsic propagation rate constant $k_{p R}$ and a diffusion term $k_{P D}$ accounting for the diffusional limitations of the propagation reaction:

$$
\frac{1}{k_{p}}=\frac{1}{k_{p R}}+\frac{1}{k_{P D}}
$$

The translational diffusion depends on the free volume of the reaction medium in the following manner:

$$
k_{P D}=D \exp \left(-\frac{g 6}{V_{f}}\right)
$$

By using the initial conditions in eqs. (18) and (19) we obtain

$$
\frac{1}{k_{p 0}}=\frac{1}{k_{p R}}+\frac{1}{k_{P D 0}}
$$

where

$$
k_{P D 0}=D \exp \left(-\frac{g 6}{V_{f 0}}\right)
$$

Substitution of eqs. (19), (20), and (21) into eq. (18) and elimination of $D$ results in

$$
k_{p}=\frac{1}{\frac{1}{k_{p 0}}+\frac{1}{k_{P D 0}}\left\{\exp \left(g 6 X_{V f}\right)-1\right\}}
$$

The glass effect function of the propagation rate can now be given according to eq. (23) by

$$
g k p=\frac{k_{p}}{k_{p 0}}=\frac{1}{1+\frac{k_{p 0}}{k_{P D 0}}\left\{\exp \left(g 6 X_{V F}\right)-1\right\}}
$$

To achieve a uniform nomenclature in our simulation model we set the adjustable model parameters as g3 $=k_{T D 0}, \mathrm{~g} 4=n$, and $\mathrm{g} 5=K_{P D O}$.

\section{Rate of Chain Transfer Reaction}

In polymer kinetics the encounter pair model has widely been applied to bimolecular termination and free radical propagation. However, the possibility that the transfer reaction at high conver- sions also will become diffusion controlled has not been properly appreciated.

The two reactions - free radical propagation and transfer to monomer-are competitive bimolecular reactions, which are supposed to require the same diffusive steps. According to this hypothesis the influence of the diffusion controlled process should be the same in both cases. Therefore, the ratio of the rate constants of the chain transfer and propagation reactions should be constant throughout the entire polymerization range. Thus, to model the rate constant for transfer to monomer $k_{t r, M}$ we can use the same type of equation as for the propagation rate constant, ${ }^{10}$ i.e., the changeover from chemical to diffusion control will occur at the same conversion for both transfer and propagations:

$$
k_{t r, M}=k_{t r, M 0} \cdot g k p
$$

We propose for the diffusion-controlled transfer reactions for transfer to chain transfer agents and solvent molecules - if the volume of the molecules are of the same orders of magnitude - the following simplified equations for transfer to chain transfer agent:

$$
k_{t r, C}=k_{t r, C 0} \cdot g k p
$$

and for transfer to solvent:

$$
k_{t r, S}=k_{t r, S 0} \cdot g k p
$$

Note that for a large difference in a molecular shape and size between the monomer molecule and the transfer agents then there may be different values for the parameters g5 and g6 in both cases.

\section{Rate of Initiation}

For modeling the change of the radical efficiency during the course of the polymerization we proposed the following empirical equation ${ }^{23}$ :

$$
f=\frac{2 f_{0}}{1+\exp \left(g 2 X_{V f}\right)}
$$

\section{RESULTS AND DISCUSSION}

In the above given model, there are six adjustable model parameters g1 to g6. The higher the number of adjustable parameters the larger will be 
the autocorrelation between them. In order to examine whether all the above model parameters have equal importance in kinetic modeling and whether all elementary processes are equally important in the model equations, we formulate the following three case studies:

1. The general Kt-Kp- $f$ model where all reactions are diffusion controlled.

2 . The $K t-K p$ model where the initiation reaction is not diffusion controlled, so that the radical efficiency remains constant over the entire conversion range, i.e., $f=f 0$.

3 . The $K t$ - $f$ model where only the termination and initiation reactions are diffusion controlled, i.e., $g k p=1$.

In the Kt- $f$ model we do not need the two adjustable parameters $g 5$ and $g 6$, while in the $K t$ $K p$ model one adjustable model parameter being g2 is not needed. We will compare these three models by adjusting the model parameters to experimental data of conversion and the average degrees of polymerization simultaneously. This systematic comparison of the influence of $f$ and $k p$ in the diffusion-controlled regime gives us information on the polymerization processes at high conversions. In the literature, various expressions describing the molecular weight dependence of self-diffusion coefficient have been reported. These expressions obey the following general relation:

$$
D_{\text {self }} \sim M^{n}
$$

Bueche $^{8}$ proposed a value for $n=3.5$ for the case of diffusion of large entangled macromolecules. Marten and Hamielec ${ }^{6}$ have used a value of 0.5 and 1.75 and assumed $M$ represents the cumulative weight-average molecular weight. In the context of the reptation theory, various investigators have proposed a value of $n=2$. For all the above three cases we have varied model parameter g4 and found g4 to be constant and independent of the reaction temperature, but depending on the type of monomer. For the polymerization of MMA and ST the values of g4 were found to be 1.0 and 0.25 , respectively. The extreme deviation of these numerical values can be explained with the very large confidence intervals that have been found for this mathematical structure. Thus, we have five, four and three adjustable parameters for the cases 1,2 , and 3 , respectively.

\section{Isothermal Polymerization of MMA ${ }^{16,17}$}

In Figure 1, model predictions of the overall adjustment of the model parameters of each model are compared with the literature data on the conversion history for the isothermal polymerization of MMA conducted with different initiator loadings. As can be seen, there is a good agreement between experimental and predicted results for all models, although at very high conversions the result of the $K t-K p$ model differs slightly from the other two. The numerical values of the model parameters with their confidence intervals and the sum of the least square errors (SLSE) are given in Table II. The general Kt-Kp- $f$ model shows the smallest value of the SLSE. The value of the model parameters $g 1, g 2$, and $g 3$ lies for all three models within their own confidence intervals, while in case of $g 5$ and $g 6$ this does not hold true. At this stage, one is not yet able to evaluate and draw conclusions to which of the models is the better one.

In the next step for the three models above simultaneous parameter estimation will be applied to conversion and degree of polymerization data for the polymerization of MMA conducted with 0.3 wt $\%$ AIBN and at $70^{\circ} \mathrm{C}$. Figure 2 give the simulated curves and the experimental data for all three models. Note that our $K t-K p$ model in this case differs from the conventional ones through the incorporation of the dependence of the transfer rate constant on conversion and that we obtain a better agreement between model and experiment than that for the Panke-SticklerHamielec model as discussed in part I. ${ }^{1}$ However, from the polymerization degree-time curve it is clear that the model predictions of the degrees of polymerization, $P_{n}$ and $P_{w}$, are lower than the experimental data, especially above $40 \%$ monomer conversion. On the contrary, the $K t$ - $f$ model shows almost the same good approach to the experimental data as that of the general model. This supports the importance of the incorporation of the change of the radical efficiency in modeling of high conversion polymerization, in case conversion and degrees of polymerization are being modeled simultaneously. It should be noted that the rate of propagation at high conversions is diffusion controlled, but that its influence on describing the conversion and degree of polymerization is lower than that of the rate of initiation. The maintaining of the radical efficiency $f$ constant over the whole range of conversion ignores an important kinetic process. Therefore, we suggest the 


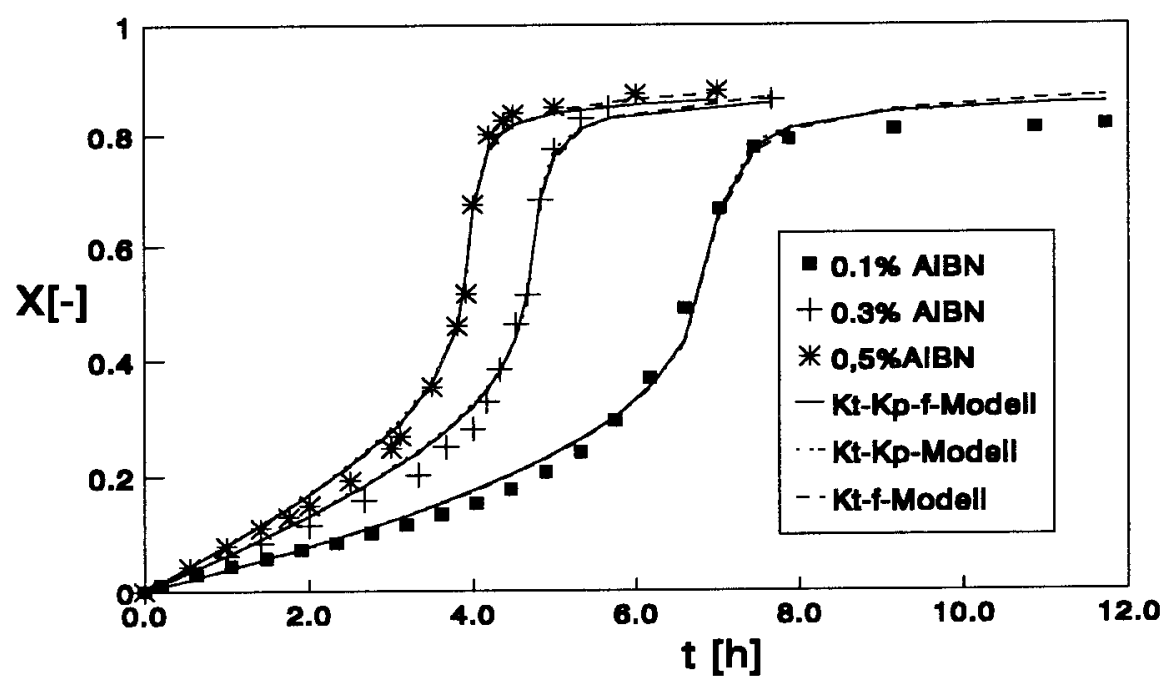

Figure 1 Comparison of models. AIBN-initiated bulk polymerization of $\mathrm{MMA}, 50^{\circ} \mathrm{C}$, fit of conversion data.

$K t-f$ model or the general $K t-K p$ - $f$ model to be used, but never the $K t-K p$ model. For the description of the MMA polymerization it seems to be enough to use the $K t$ - $f$ model, which contains only three adjustable parameters.

From a mathematical point of view, confidence interval (CI) analysis can also serve as a criterion for the model selection. The CIs of the parameters of the $k t-k p$ - $f$ and the $k t-k p$ model are very large and in the same order of magnitude. Contrarily, the CIs of the $k t$ - $f$ model are only about $30 \%$ of the both other models. This shows that reducing the number of adjustable parameters can ex- tremely improve the model if "nonimportant" kinetic steps are neglected. This also leads to the conclusion that the $k p$ correlation seems to be "nonimportant."

For the modeling of the polymerization of MMA we select the $K t$ - $f$ model. As a next step from isothermal experiments conducted at different temperature of $50-90^{\circ} \mathrm{C}$ we determine the temperature dependencies of the model parameters by simultaneous parameter estimation applied to the conversion and degrees of polymerization data. The numerical values obtained of the adjusted parameters are given in Table III. Both $g 1$ and g3

Table II Parameters Estimation on the Basis of a Monomer Conversion Data Set of an AIBN-Initiated Polymerization of MMA at $50^{\circ} \mathrm{C}$

\begin{tabular}{|c|c|c|c|}
\hline Model & Parameter & Value & $\begin{array}{c}\text { Sum of the } \\
\text { Least Square } \\
\text { Errors (SLCE) }\end{array}$ \\
\hline \multirow[t]{5}{*}{$k t-k p-f$-Model } & g1 & $1.637 \pm 0.102$ & \multirow[t]{5}{*}{$2.536 \times 10^{-2}$} \\
\hline & g2 & $0.342 \pm 0.047$ & \\
\hline & g3 & $\exp (31.999 \pm 0.158)$ & \\
\hline & g5 & $\exp (11.471 \pm 1.559)$ & \\
\hline & g6 & $0.287 \pm 0.027$ & \\
\hline \multirow[t]{4}{*}{$k t$ - $k p$-Model } & g1 & $1.549 \pm 0.174$ & \multirow[t]{4}{*}{$2.746 \times 10^{-2}$} \\
\hline & g3 & $\exp (31.994 \pm 0.177)$ & \\
\hline & g5 & $\exp (9.685 \pm 1.455)$ & \\
\hline & g6 & $0.540 \pm 0.096$ & \\
\hline \multirow[t]{3}{*}{$k t$-f-Model } & g1 & $1.676 \pm 0.121$ & \multirow[t]{3}{*}{$2.780 \times 10^{-2}$} \\
\hline & g2 & $0.379 \pm 0.023$ & \\
\hline & g3 & $\exp (32.036 \pm 0.159)$ & \\
\hline
\end{tabular}


(a)

(b)
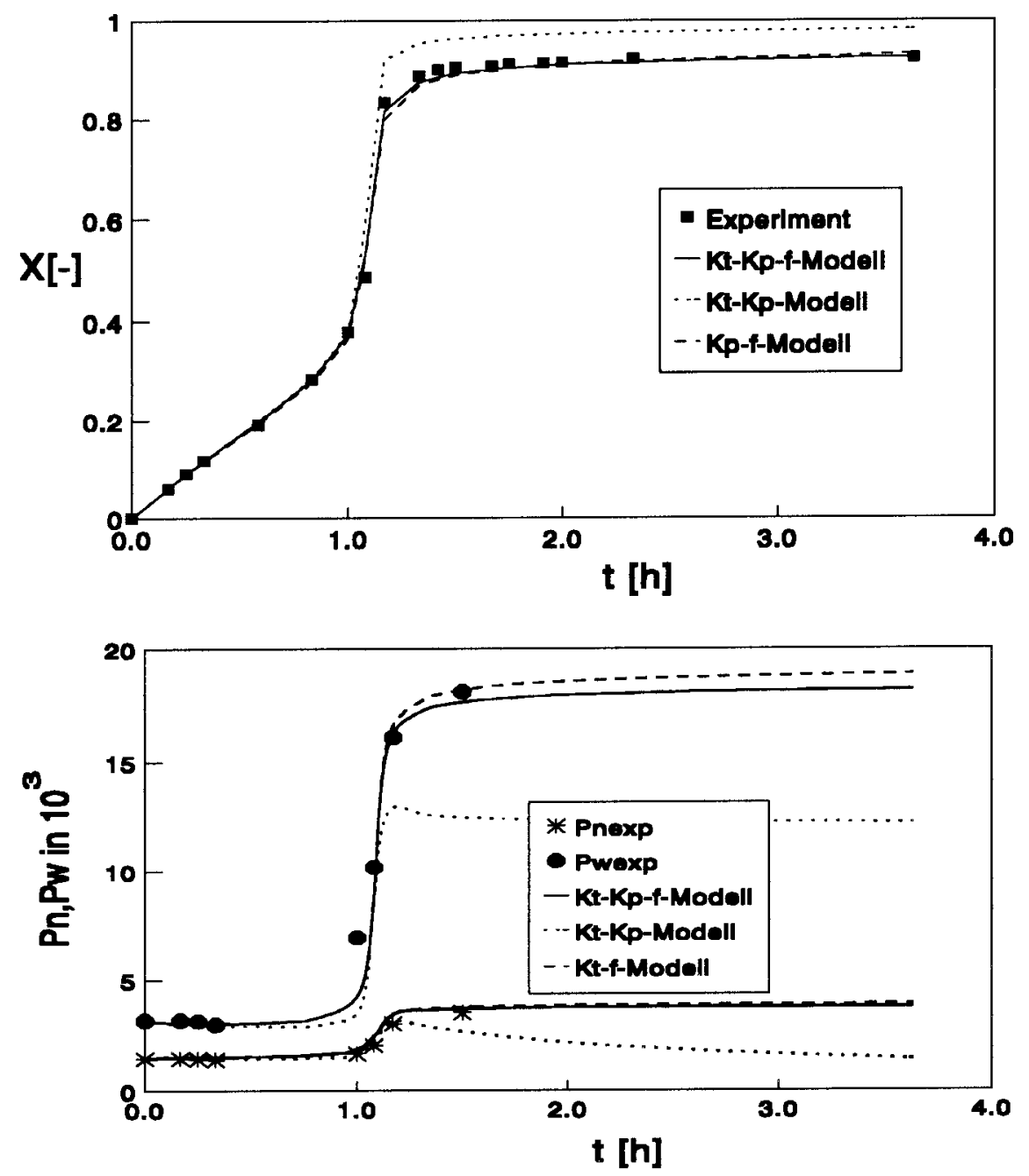

Figure 2 Comparison of models. AIBN-initiated bulk polymerization of MMA, 0.3 wt $\%$ AIBN, $70^{\circ} \mathrm{C}$, simultaneous fit of conversion and average degree of polymerization data: (a) monomer conversion, (b) degree of polymerization.

are parameters for describing the termination rate constant $k_{t}$, whereas $g 2$ is used for the rate of initiation, i.e., to describe $f$. $g 1$ appears to be

Table III Results of the Simultaneous Parameter Estimation Using the $K t$ - $f$-Model at Different Reaction Temperatures for the Polymerization of MMA

\begin{tabular}{cccc}
\hline$T\left({ }^{\circ} \mathrm{C}\right)$ & $\mathrm{g} 1$ & $\mathrm{~g} 2$ & $\mathrm{~g} 3$ \\
\hline 50 & 1.6755 & 0.37885 & $8.186 \times 10^{13}$ \\
70 & 1.9304 & 0.42807 & $7.377 \times 10^{13}$ \\
90 & 1.8703 & 0.48874 & $3.802 \times 10^{13}$ \\
\hline
\end{tabular}

temperature independent and constant, whereas g2 increases with the reaction temperature. We have used the mean value of $g 1=1.8254$ throughout this work. For g2 the following temperature dependence was obtained:

$$
g 2=3.792 \times \exp \left(\frac{-746}{T}\right)
$$

With these parameters set we adjusted the value of g3 again for the above experiments. In general, g3 decreases with temperature. However, this temperature dependence is small, very low, and gives the following Arrhenius temperature dependence is found: 


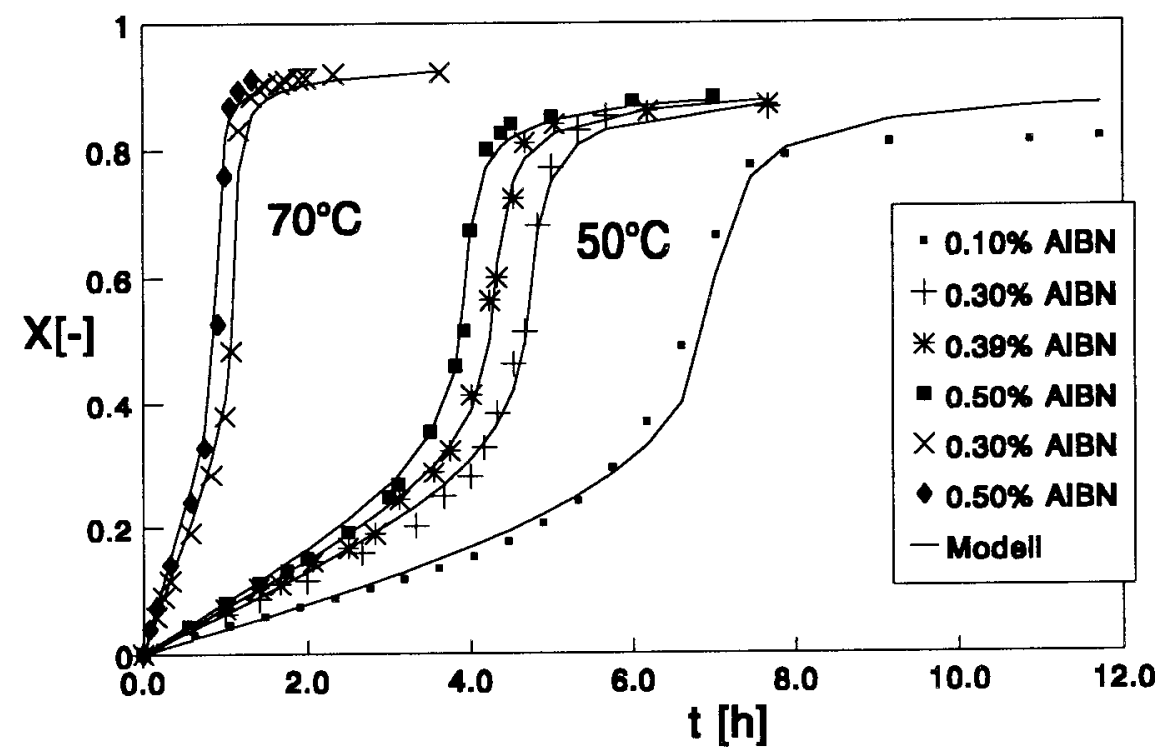

b)

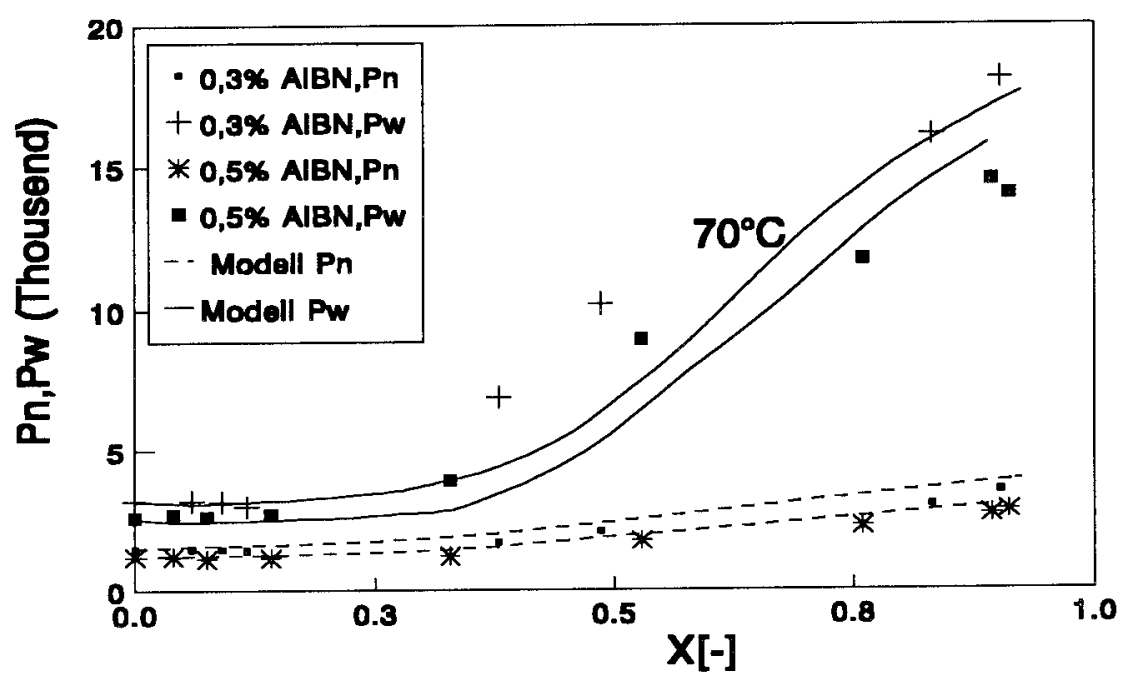

Figure 3 Comparison of model ( $K t$ - $f$ model) and experiment. AIBN-initiated isothermal bulk polymerization of MMA, 0.1-0.5 wt \% AIBN: (a) monomer conversion, (b) degree of polymerization.

$$
g 3=5.101 \times 10^{9} \exp \left(\frac{3211}{T}\right)
$$

Figure 3 compares simulation and experimental results of the conversion and degrees of polymerizations. Figure 4 also shows the comparison of the calculated and experimental MWD for the suspension polymerization of MMA conducted with 0.3 wt $\%$ AIBN at $70^{\circ} \mathrm{C} .^{18}$ These results show much better agreement between model and experiment than for the earlier proposed models.

\section{Isothermal Polymerization of Styrene}

Following the above procedure the model parameters were fitted for the suspension polymerization of styrene with AIBN conducted at different temperatures of 70,75 , and $80^{\circ} \mathrm{C}$. The experimental 


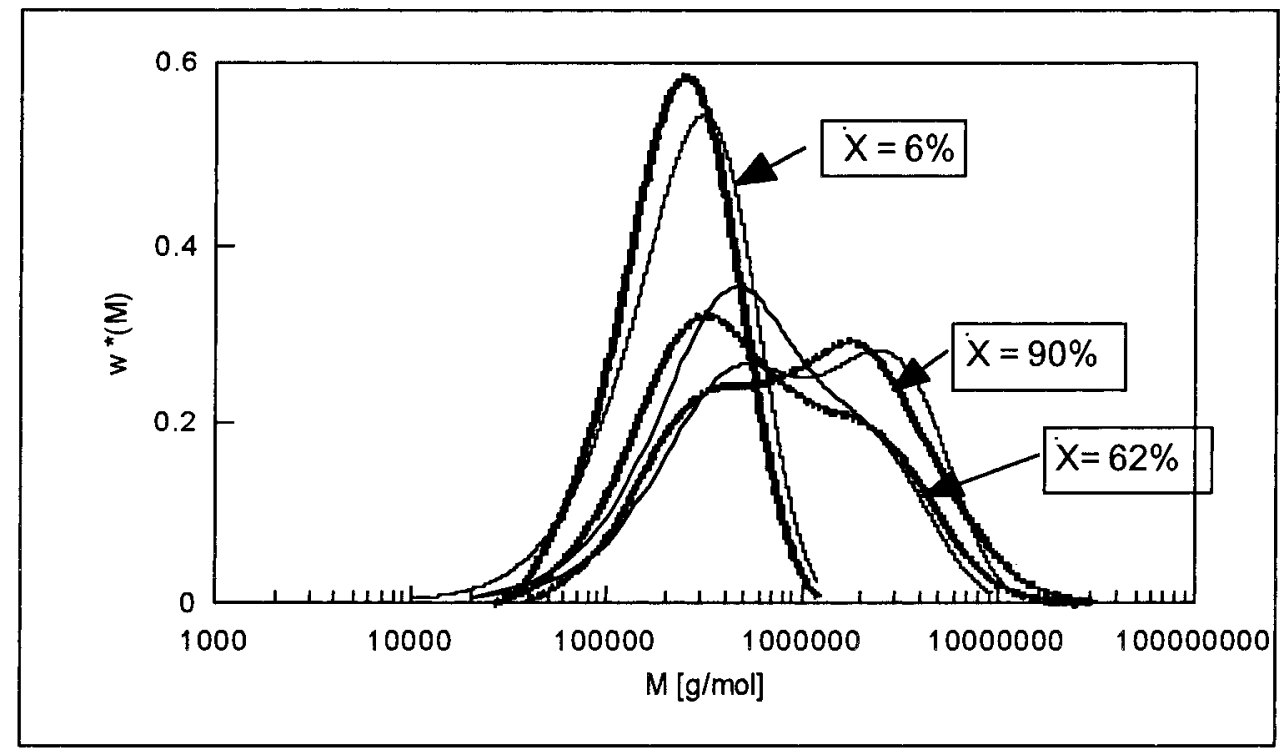

Figure 4 Comparison of model (thin line) and experiment (thick line). Suspension polymerization of MMA, $0.3 \mathrm{wt} \%$ AIBN. Molecular weight distribution at different monomer conversion. $w^{*}=f(\log M)$.

conversion and degree of polymerization data were determined using a GPC as mentioned elsewhere.$^{14}$ Using the $K t$ - $f$ model, the simulated conversion-time curve differs from the experimental one, especially at very high conversions, while for the degrees of polymerization prediction and experiment are in good agreement. This may indicate that the $K t$ - $f$ model may not be able to describe these experimental results. Therefore, we use the general model in order to correct for these discrepancies. The result of fitted parameters are now

$$
\begin{aligned}
& g 1=0.32 ; g 2=0.148 ; \quad g 3=1.4382 \times 10^{9}, \\
& g 5=9.14 \times 10^{4} \quad \text { and } \quad g 6=0.21
\end{aligned}
$$

The comparison of simulated and experimental results is shown in Figure 5. There is a good agreement between model and experiment. For this temperature range the parameters are not significantly dependent on temperature.

For the thermally initiated polymerization of styrene $^{15}$ in the temperature range of 100 to $200^{\circ} \mathrm{C}$ above the gals transition temperature of polystyrene of $\operatorname{Tgp}=100^{\circ} \mathrm{C}$ it was found that the parameters $g 1=0.32$ and $g 3=1.4 \times 10^{9}$ describe the conversion-time histories well, despite small differences in the degrees of polymerization in Figure 6.

The above results show that the presented model describes well the thermally and AIBN-initiated isothermal bulk and suspension polymerization of styrene and MMA, conducted in the temperature ranges of $70-200^{\circ} \mathrm{C}$ and $50-90^{\circ} \mathrm{C}$, respectively.

\section{Application to Bulk and Suspension Polymerization of MMA}

For the industrial application it is very important to investigate the flexibility of the model towards varying operational conditions. Among the many operational variables in polymerization reaction engineering variation of the type of initiator, the use of a chain transfer agent CTA, and/or a solvent and the reaction temperature are the most important ones to control the polymerization process and the product quality. In this section results are presented for a change of the type of initiator and the concentration of the CTA.

\section{Results on Lauryl Peroxide (LPO)-Initiated Bulk Polymerization of MMA}

In general, for a polymerization conducted by only changing the type of the initiator, it is expected that only the specific parameters of the initiator, $\mathrm{k}_{d}, f 0, g 2$, will be different in the kinetic equations. For the LPO-initiated polymerization of MMA the dissociation constant $k_{d}$ is given by ${ }^{17}$ 
a)

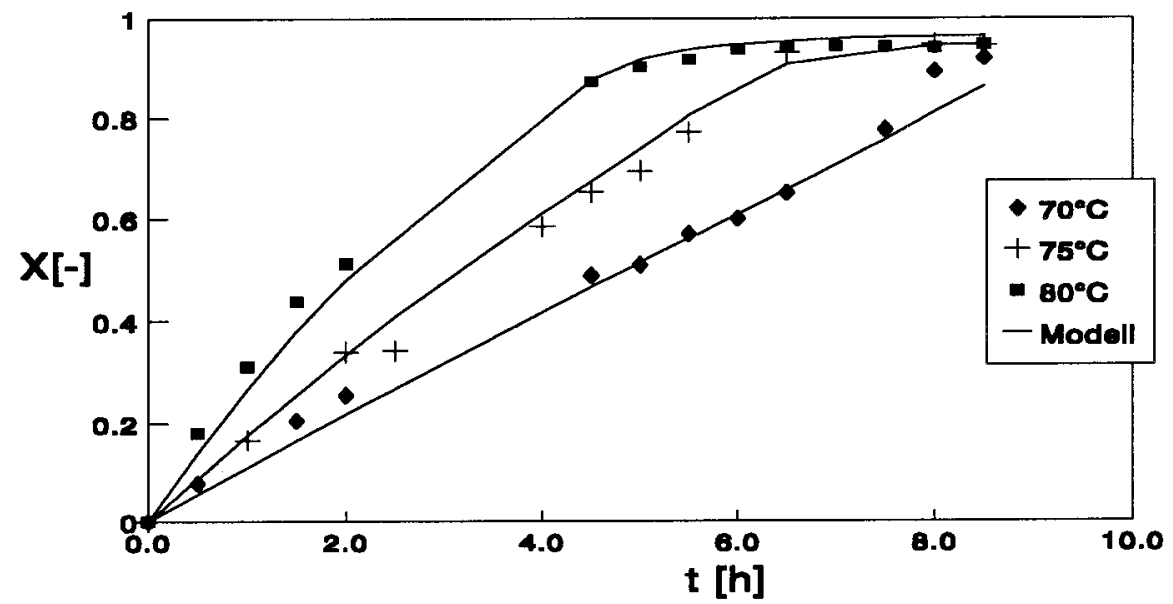

b)

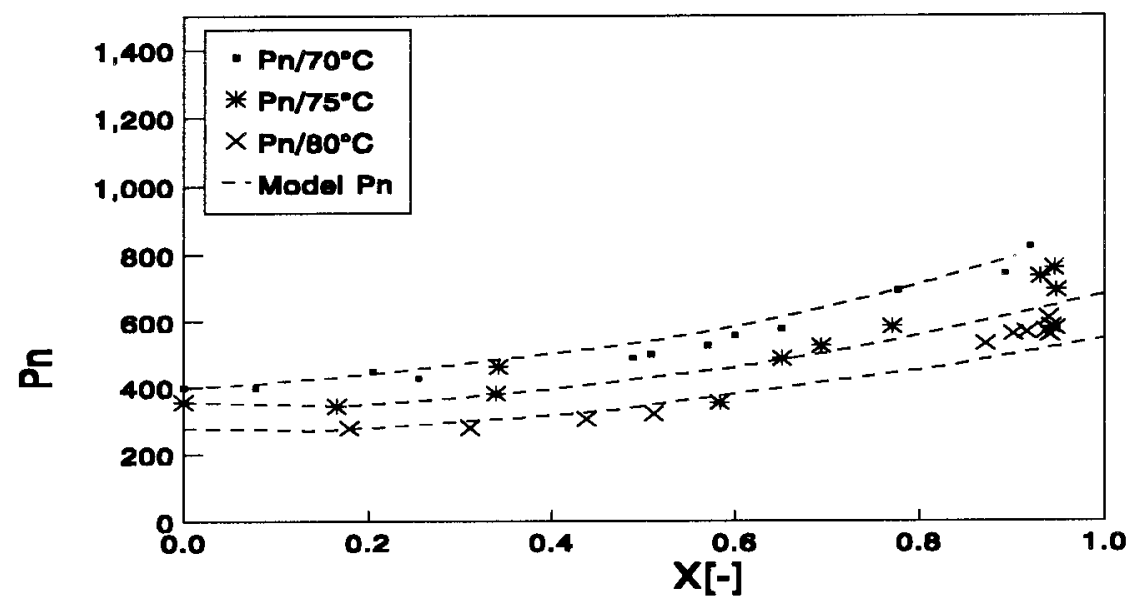

c)

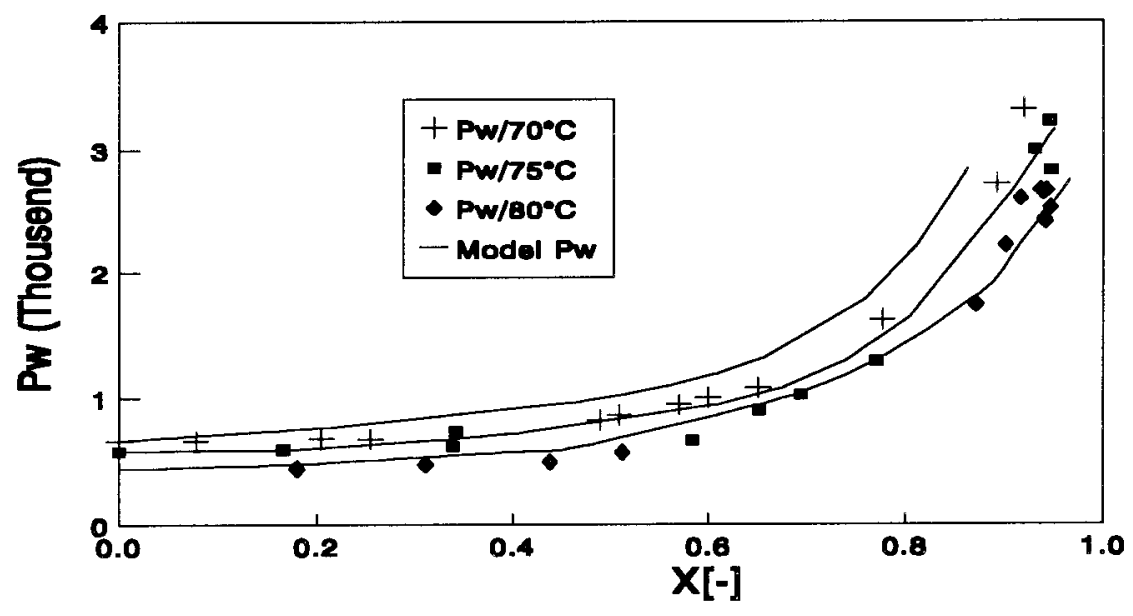

Figure 5 Comparison of model ( Kt-Kp- $f$ model) and experiment. ${ }^{14}$ Suspension polymerization of styrene, $0.45 \mathrm{wt} \%$ AIBN: (a) monomer conversion, (b) number average degree of polymerization, (c) weight average degree of polymerization. 
(a)

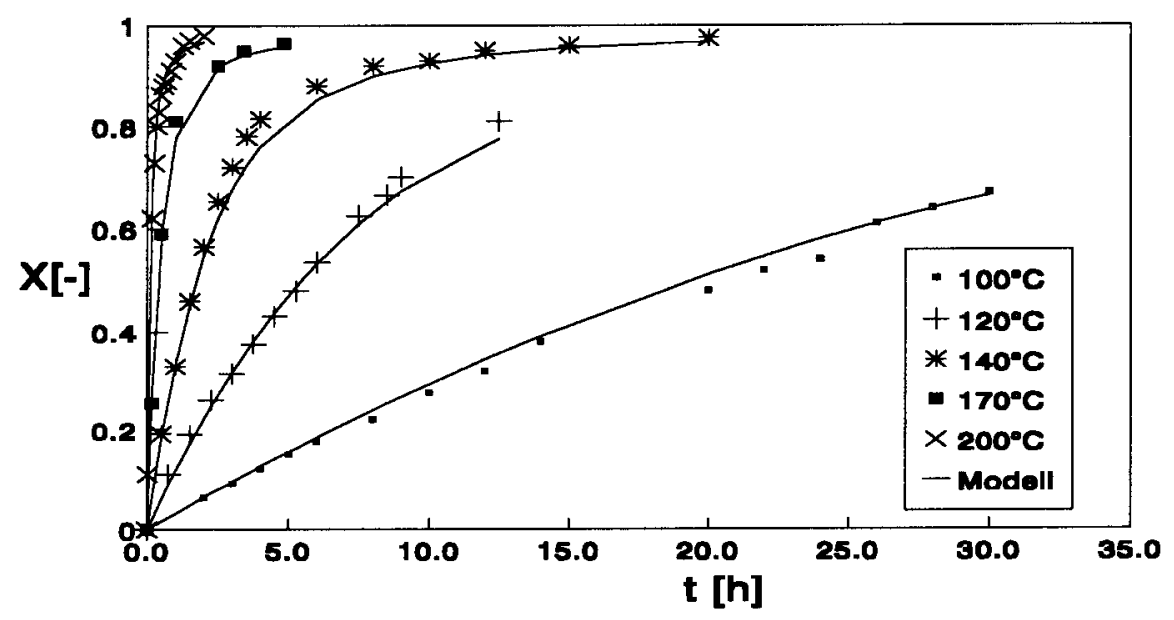

(b)

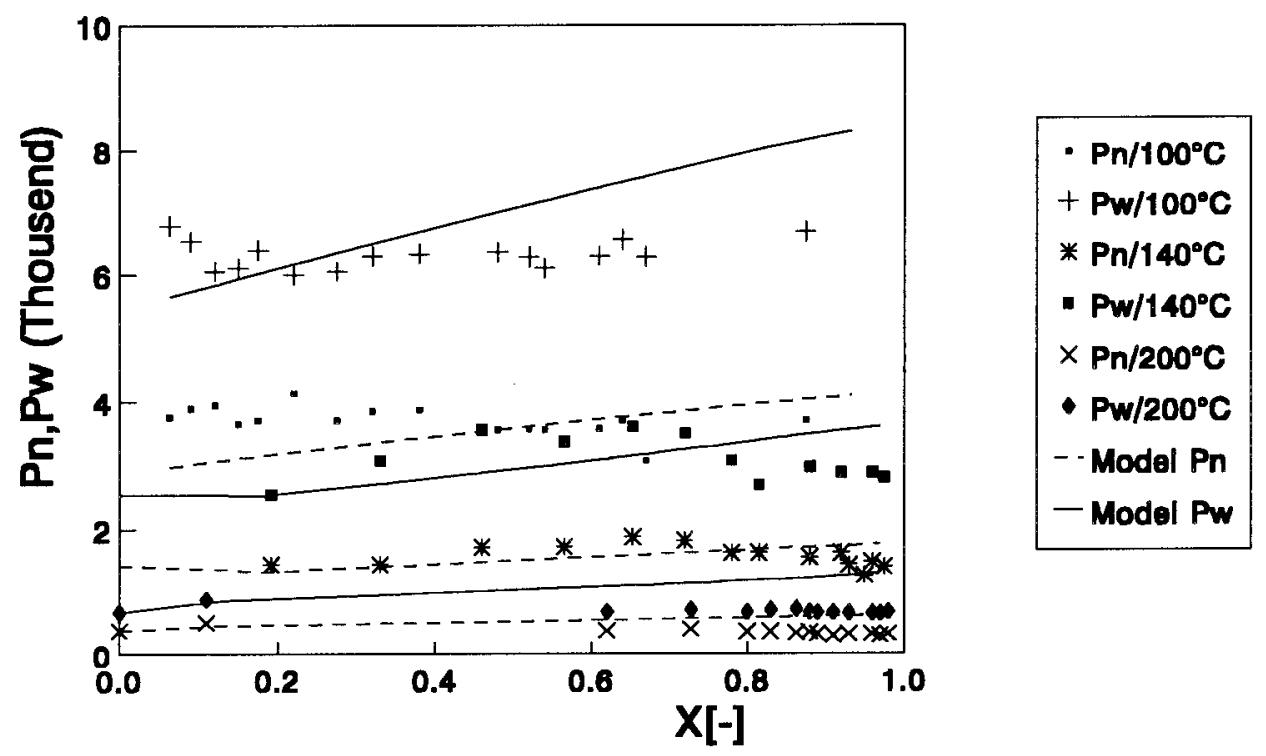

Figure 6 Comparison of model ( Kt-Kp- $f$ model) and experiment. ${ }^{14}$ Thermally initiated polymerization of styrene: (a) monomer conversion, (b) number- and weightaverage degrees of polymerization $(P n, P w)$.

$$
k_{d}=1.351510^{15} \exp \left(\frac{-15400}{T}\right) s^{-1}
$$

Using the initial kinetic constants given in Table I the radical efficiency $F 0$ is estimated on the basis of initial time-conversion data to be $f 0$ $=0.4$. For the calculation of the variation of $f$ with conversion, the parameter $g 2$ should be estimated from the time-conversion data over the whole conversion range. The simultaneous parameter estimation on the basis of the time-conversion data at $50^{\circ} \mathrm{C}$ and with different initiator loadings of $0.1-1.0$ wt $\%$ gives

$$
g 2=0.46
$$

with a confidence interval of $13.4 \%$. In Figure 7 the monomer conversion is plotted against reaction time for different LPO concentrations. As can be seen, the experimental and simulated results are in rather good agreement within the experimental error. Note that the monomer conversion 


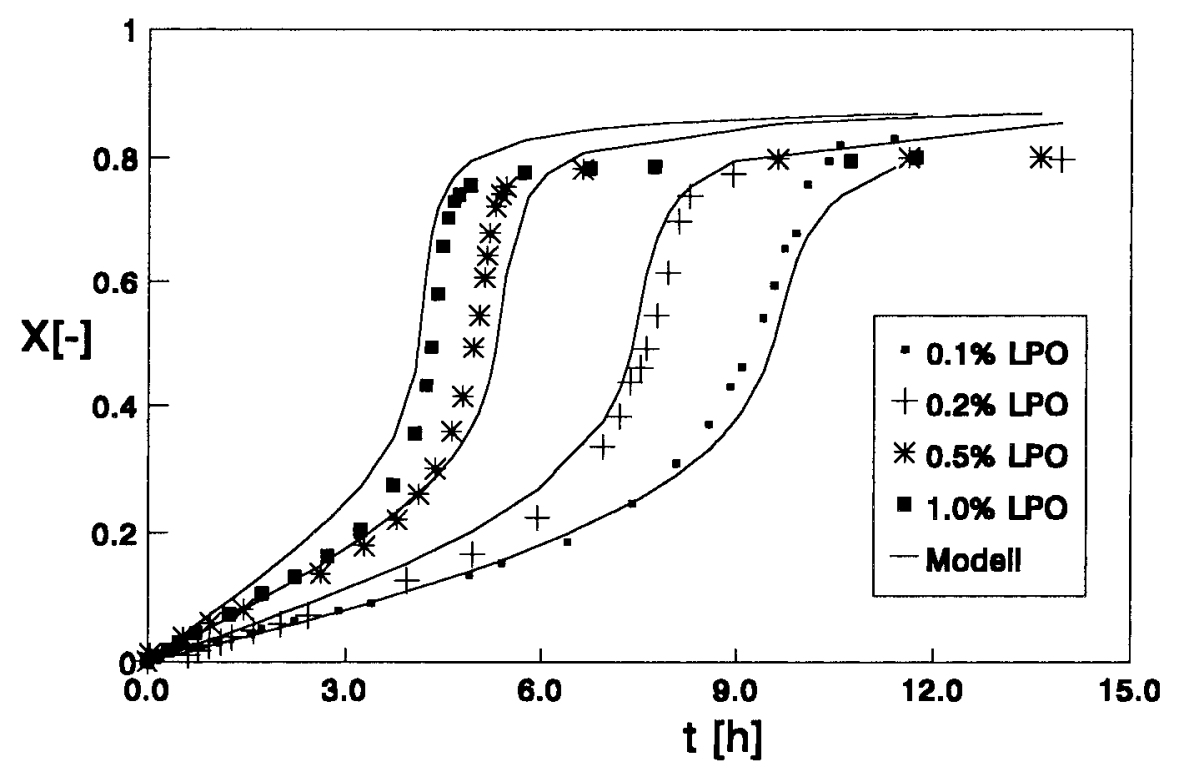

Figure 7 Application to another initiator type. LPO-initiated bulk polymerization of MMA conducted at $50^{\circ} \mathrm{C}$. Monomer conversion (by dilatometry). ${ }^{17}$

was determined by dilatometry. ${ }^{17}$ At the initial stage of polymerization there is no difference between the experiments conducted with 0.1 and 0.2 , as well as 0.5 and $1.0 \mathrm{wt} \%$ LPO. This discrepancy most likely is due to the large experimental error at low conversions. The value of g2 for LPO is greater than that for AIBN. This may be due to the fact that the molar volume of the LPO radical is larger than that of the AIBN radical; consequently, the AIBN radical can diffuse faster out of the cage. This shows us the only parameters to be reestimated for a diffusion-controlled polymerization is the initial radical efficiency and $g 2$, if the initiator is changed for the same polymerization.

\section{The AIBN-Initiated Polymerization of MMA with a CTA}

Chain transfer agents (CTAs) affect not only the molecular weight of the resulting polymer but also the magnitude and the onset of the gel effect. It has been pointed out that an increase in the concentration of the CTA delays the onset of the gel effect and reduces its magnitude. Moreover, the presence of a CTA in the system may modify the final conversion of the polymer obtained. ${ }^{22}$

The ability of the presented model to predict monomer conversion and molecular weight development for the bulk and suspension polymerization of MMA in the presence of a CTA (dodecyl mercaptan, DDM) at the beginning of the reaction is demonstrated in Figures 8-11. Figure 8 illustrates the variation of the monomer conversion with time and the weight-average degree of polymerization against monomer conversion for the AIBN-initiated bulk polymerization of MMA, conducted at $70^{\circ} \mathrm{C}$ at different concentrations of CTA of $0.2-0.8 \mathrm{wt} \%$ as well as the corresponding calculations. The monomer conversion was determined gravimetrically and the MWD of the polymer by GPC. The predicted and experimental values of the conversion agree within the experimental error. The experimental and simulated results of the average degree of polymerization also agree well for low concentrations of the CTA, but starts to deviate at increasing concentrations of CTA. This may be due to fact that the low molecular weight polymer is not completely isolated using a gravimetrical method. The polymer, isolated by precipitation, has a higher molecular weight than the polymer without precipitation. Furthermore, the monomer conversion obtained by precipitation is lower, because the low molecular weight species remain in solution.

In case of the suspension polymerization the monomer conversion and MWD were determined by GPC as mentioned elsewhere. ${ }^{18}$ In Figure 9, the monomer conversion against time and the average degree of polymerization against conversion are plotted for the isothermal polymerization of MMA at $T=70^{\circ} \mathrm{C}$ and conducted at different CTA concentrations. The agreement between experi- 
a)

b)
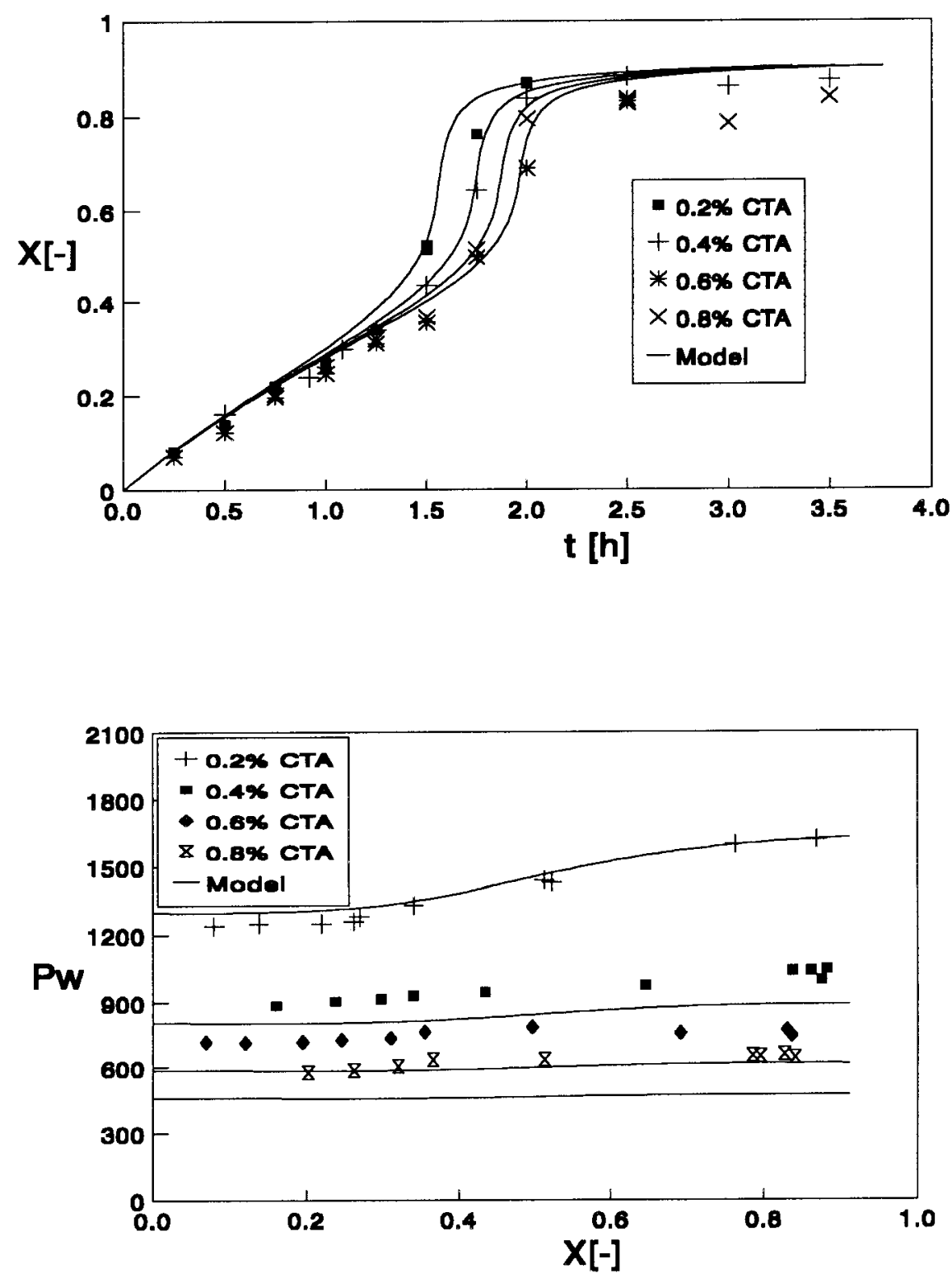

Figure 8 Use of chain transfer agent. Bulk polymerization of MMA, $70^{\circ} \mathrm{C}, 0.3 \mathrm{wt} \%$ AIBN; CTA, dodecyl mercaptan. Monomer conversion (by gravimetry) and degree of polymerization.

ment and model is good for the monomer conversion and the average degree of polymerization.

To test the applicability of the presented model to semibatch reactor operation, $0.4 \mathrm{wt} \%$ of CTA were injected during a suspension polymerization of MMA at different reaction times. ${ }^{18}$ In Figure 10 both experimental and predicted results are shown. For the injection of CTA at $t=0$ there is a good agreement between experiment and model. If the moment of injection increases, the discrep- ancy in the degree of polymerization between experiment and simulation increases. An explanation could be given as follows. During the course of the polymerization the viscosity of the reaction mixture in the particle increases, which leads to a reduction of the diffusivity of the CTA from the water phase to the organic phase and the concentration of the CTA in the organic phase becomes relatively low. As a result, polymers with a high molecular weight will be produced. In the pre- 
a)

b)
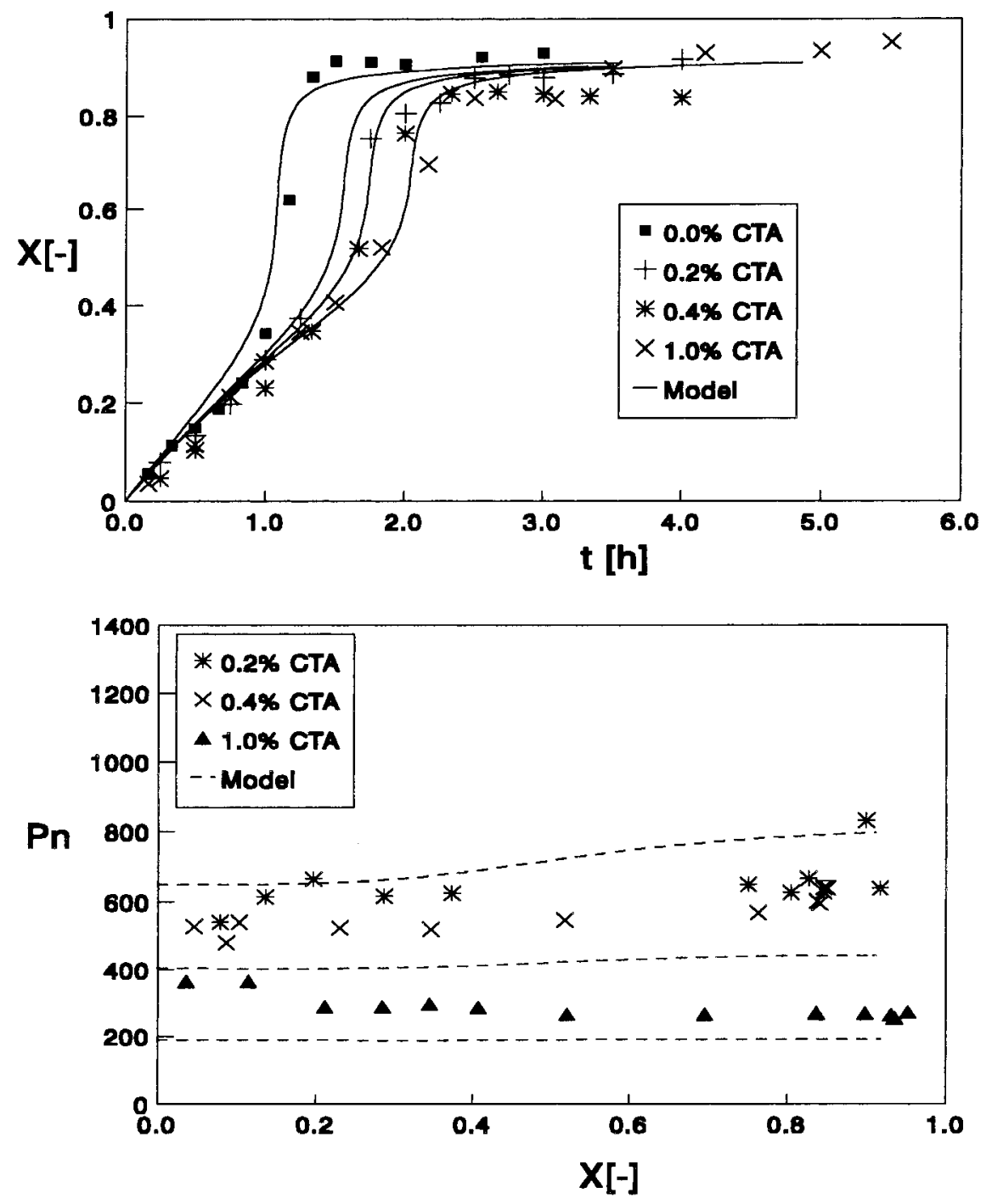

c)

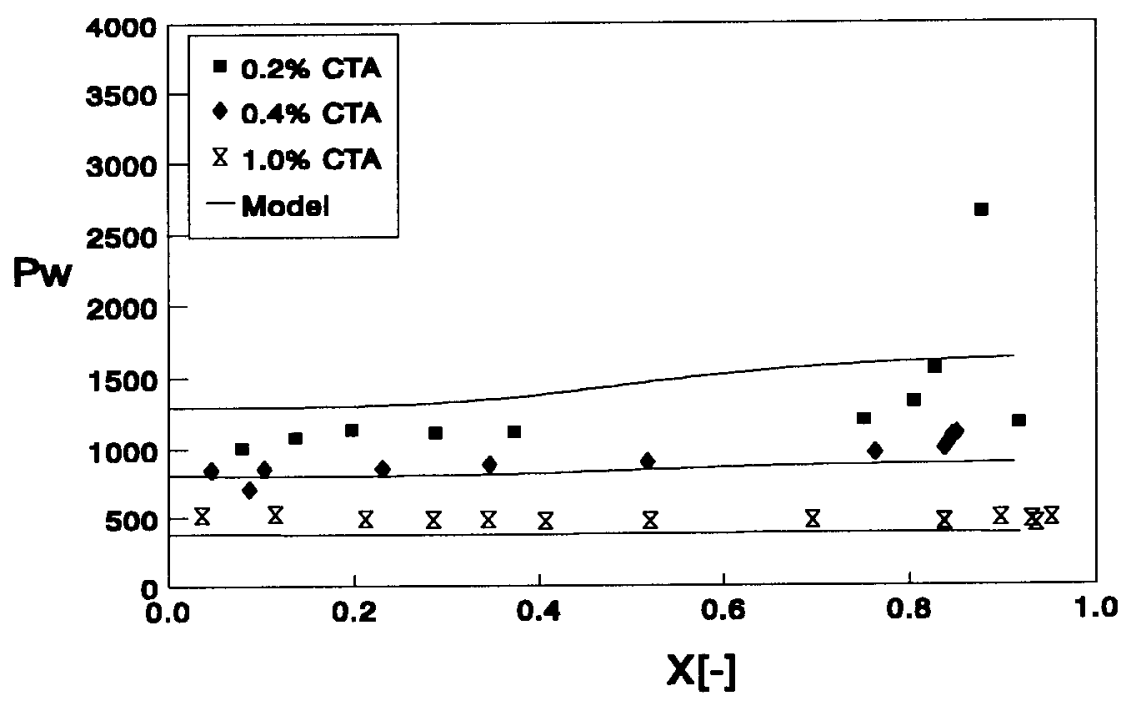

Figure 9 Use of chain transfer agent. Suspension polymerization of MMA, 70 ${ }^{\circ} \mathrm{C}, 0.3$ wt \% AIBN; CTA, dodecyl mercaptan. Monomer conversion (by GPC) and degree of polymerization. 
a)

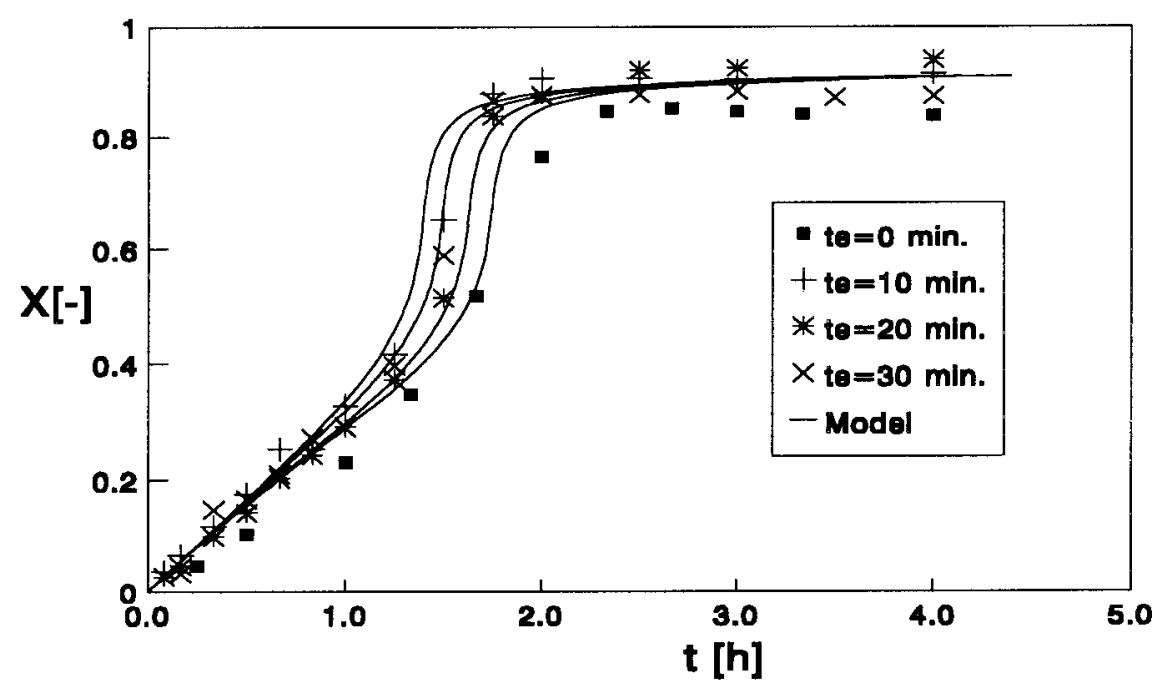

b)

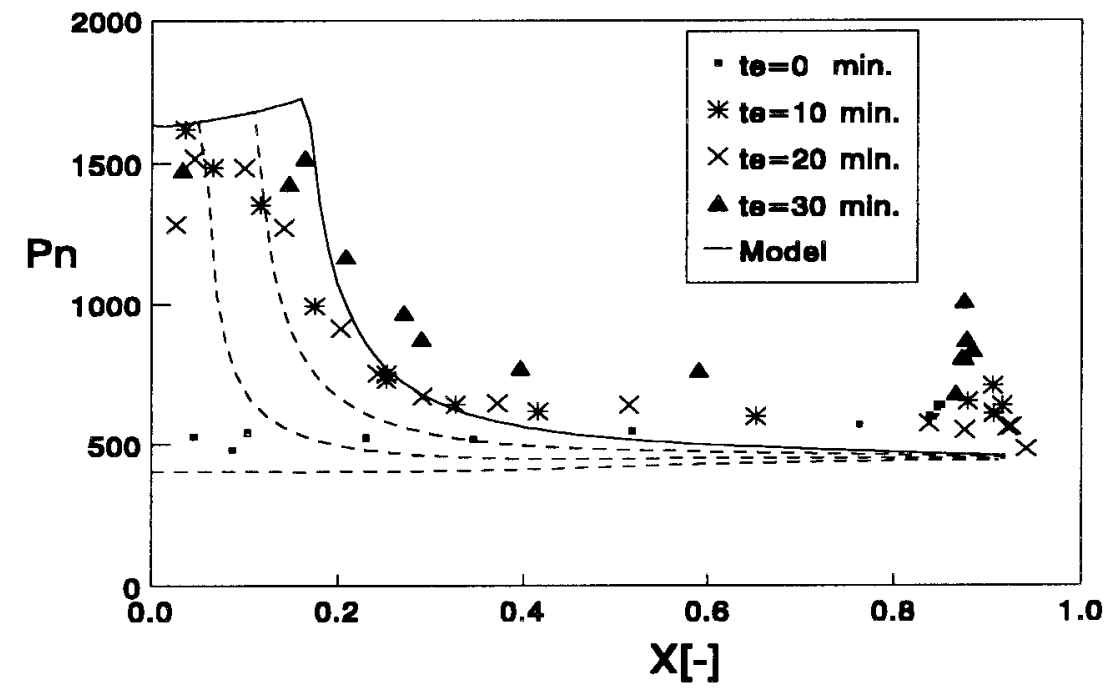

c)

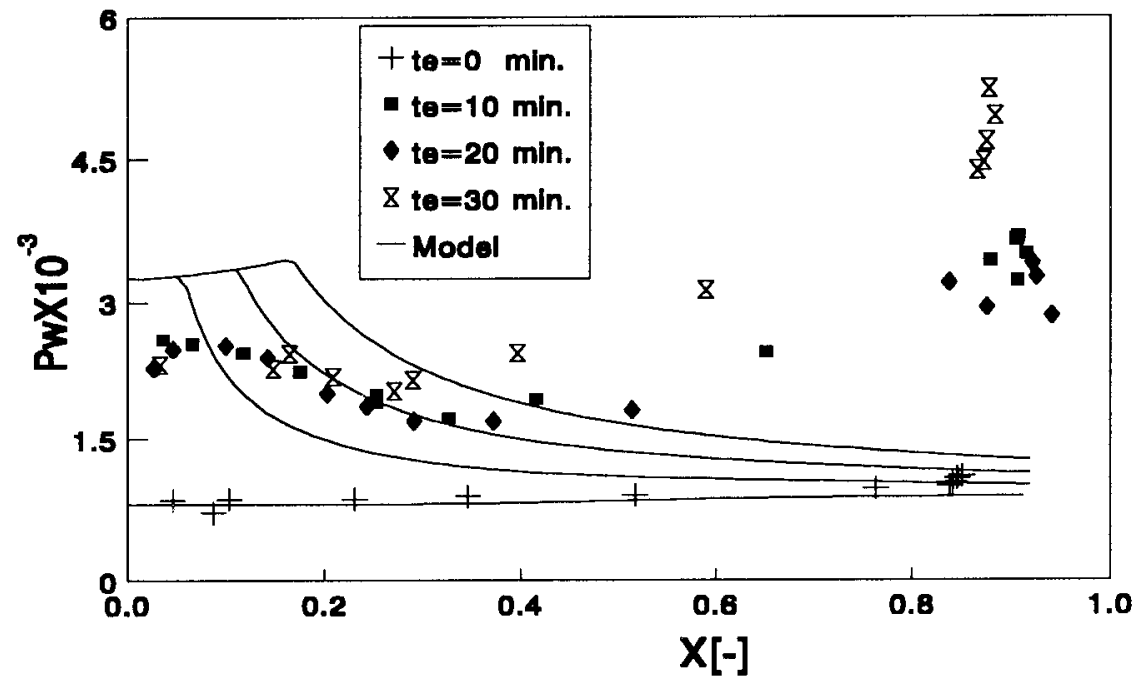

Figure 10 Influence of the injection time of CTA $(t e)$ on the average degree of polymerization. Suspension polymerization of MMA, $0.3 \mathrm{wt} \%$ AIBN, $0.4 \mathrm{wt} \% \mathrm{DDM}, T=70^{\circ} \mathrm{C}$. Monomer conversion (by GPC) and degree of polymerization. 
a)

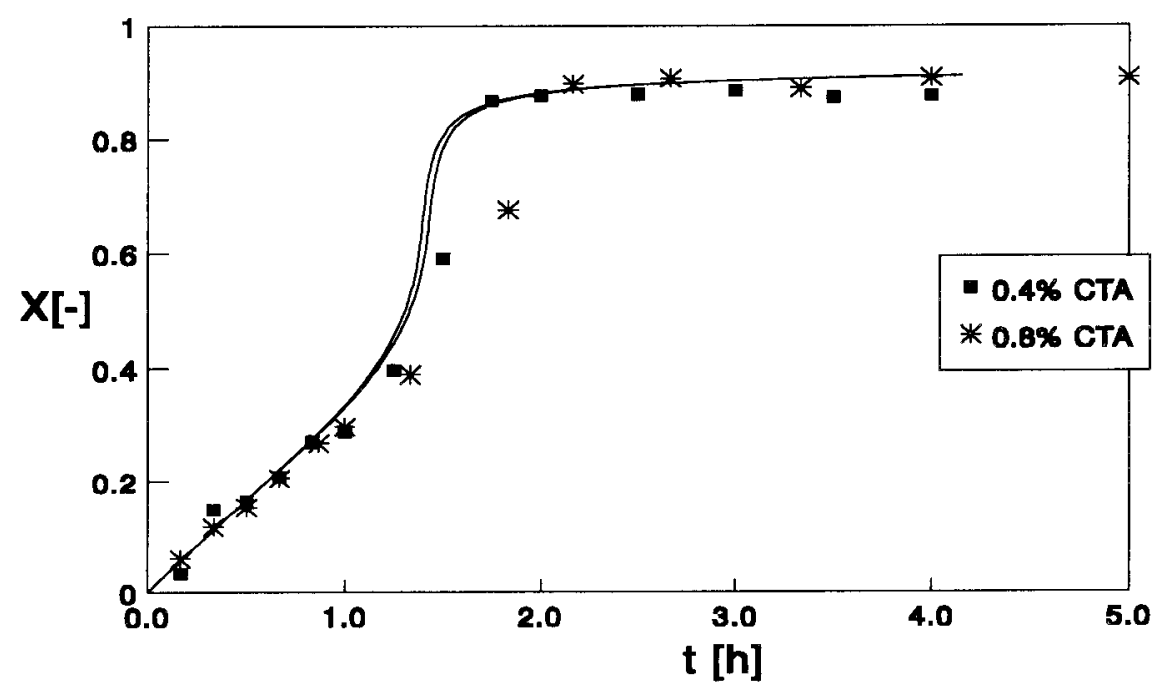

b)

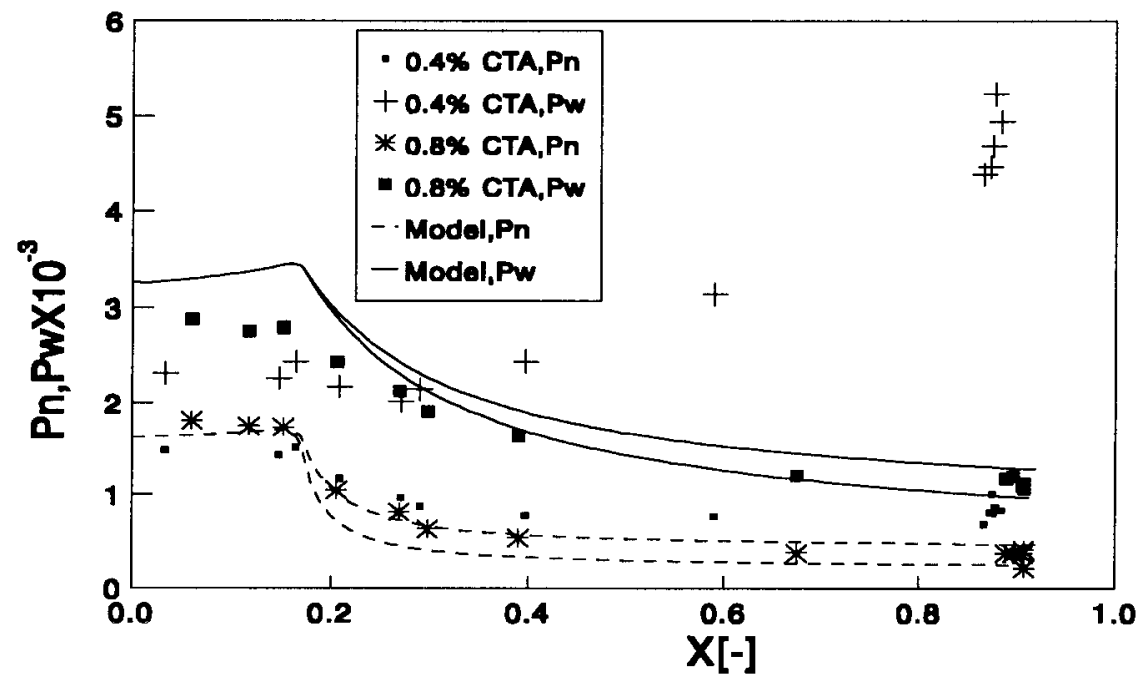

Figure 11 Influence of the concentration of CTA at $t e=0.5 \mathrm{~h}$ on the average degree of polymerization. Suspension polymerization of MMA, $70^{\circ} \mathrm{C}, 0.3$ wt $\%$ AIBN. Monomer conversion (by GPC) and average degrees of polymerization.

sented reactor model this mass transfer effect has not been implemented. To demonstrate this the concentration of the CTA was doubled to $0.8 \mathrm{wt}$ $\%$ at the moment of injection of $30 \mathrm{~min}$. At this point it was expected that the increasing concentration of the CTA in the water phase should accelerate the mass transfer rate due to an increasing driving force, leading to the formation of polymers with shorter chain lengths. Figure 11 clearly shows this effect. In this case, both the experimental and simulated average degree of polymerization are in good agreement. Other applications of the presented model, for example, for nonisothermal polymerization processes, for the optimization of the MWD and for comparison with data from calorimetric measurements, are discussed elsewhere. $^{18}$

\section{CONCLUSION}

We have developed a semiempirical model for the description and prediction of free radical chain polymerization processes. This model incorporates the diffusion processes from the beginning of the reaction; hence, it does not require the introduction of artificial break points for diffusion limitations. The model also incorporates the change of all rate parameters due to the increase of conversion. It has been shown that the change 
of the radical efficiency at high conversions is more important than the change of the propagation rate constant. For modeling the polymerization of MMA the $K t$ - $f$ model may suffice, whereas for styrene, the general model should be considered. The model parameters are linked to operating variables of the process, in particular to the temperature of the system. The simulated values of conversion, polymerization degree and MWD are in good accordance with experimental data for the suspension and bulk polymerization of MMA and ST up to very high conversions.

Financial support from the Friedrich-Naumann Foundation (Germany) is greatly appreciated.

\section{REFERENCES}

1. N. Tefera, G. Weickert, and R. K. Westerterp, J. Appl. Polym. Sci., 63, 1649 (1997).

2. W. Y. Chiu, G. M. Carratt, and D. S. Soong, Macromolecules, 16, 348 (1983).

3. D. Achilias and C. Kiparissides, Macromolecules, 25, 3739 ( 1992 ).

4. M. Buback, Makromol. Chem., 191, 1575 (1991).

5. G. V. Schulz, Z. Phys. Chem., 8, 290 (1956).

6. F. L. Marten and A. E. Hamielec, Am. Chem. Soc., Symp. Ser., 104, 43 (1979).

7. M. Smoluchowski, Z. Phys. Chem., 92, 129 (1918).
8. F. Bueche, Physical Properties of Polymers, Interscience, New York, 1962.

9. G. T. Russell, D. H. Napper, and R. G. Gilbert, Macromolecules, 21, 2133 (1988).

10. S. K. Soh and D. C. Sundberg, J. Polym. Sci., Polym. Chem. Ed., 20, 1299, 1315 (1982).

11. B. S. Casey, M. F. Mills, D. F. Sangster, R. G. Gilbert, and D. H. Napper, Macromolecules, 25, 7063 (1992).

12. G. Weickert and N. Tefera, lecture on the IUPAC Working Party, Polymerization Reactor Engineering, proceedings, Nancy, 1991.

13. G. Weickert and N. Tefera, in Proceedings of 4 th International Workshop on Polymer Reaction Engineering, K.-H. Reichert and H.-U. Moritz, Eds., Verlag Chemie, Weinheim, 1992, p. 115.

14. N. Tefera, G. Weickert, R. Bloodworth, and J. Schweer, Macromol. Chem. Phys., 195, 3067 (1994).

15. A. W. Hui and A. E. Hamielec, J. Appl. Polym. Sci., 16, 749 (1972).

16. S. T. Balke and A. E. Hamielec, J. Appl. Polym. Sci., 17, 905 (1973).

17. D. Panke, Röhm AG, Personal information.

18. N. Tefera, Ph.D. thesis, University of Twente (June 1995 ).

19. K. Horie, I. Mita, and M. Kabe, J. Polym. Sci., Chem., A1 (6), 2663 (1968).

20. K. Mahabadi and K. F. O'Driscoll, J. Macromol. Sci., Chem., A11(5), 967 (1977).

21. G. Weickert, Modellierung von Polymerisationsreaktoren, Springer, Berlin, 1996, to appear.

22. E. L. Madruga, J. J. Malfeito, Eur. Polym. J., 28(8), 863 (1992).

23. N. Tefera, Ph.D. thesis, Twente University (1995). 